\title{
Digital filters windowing for data transmission enhancement in communication channel
}

\author{
Ola N. Kadhim ${ }^{1}$, Kifah T. Khudhair ${ }^{2}$, Fallah H. Najjar ${ }^{3}$, Hassan M. Al-Jawahry ${ }^{4}$ \\ ${ }^{1}$ Technical Institute of Al-Mussaib, Al-Furat Al-Awsat Technical University, Najaf, Iraq \\ ${ }^{2,3,4}$ Technical College of Management/Kufa, Al-Furat Al-Awsat Technical University, Kufa, Iraq
}

\begin{tabular}{l}
\hline \hline Article Info \\
\hline Article history: \\
Received Feb 16, 2021 \\
Revised Oct 29, 2021 \\
Accepted Nov 2, 2021 \\
\hline
\end{tabular}

\section{Keywords:}

Data correction

Digital filter design

Digital modulation

Equalization

Filter window

\begin{abstract}
In this search, an important methodology has been presented for communicated information rectification utilizing advanced channel windowing approach. The modern data communication technologies are ensured with numerous challenges because of their unpredictability and arrangement. Various digital transmission topologies in $4 \mathrm{G}$ can't fulfill the requirements in future arrangements, therefore, alternative multicarrier modulation (MCM) becoming the nominated approaches among all other data transmission techniques. Wherein prototype filter configuration is a fundamental system based on which the synthesis and analysis filters are derived. This paper presents a complete review on the ongoing advances of finite impulse response (FIR) filter plan procedures in MCM based correspondence frameworks. Initially, the essential issues are tried, taking into consideration the presentation of available data signal applicants and the FIR filter design concept. At that point the techniques for FIR filter configuration are summed up in subtleties and are center around the accompanying three group's recurrence testing strategies, windowing based strategies and advancement-based techniques. At last, the exhibitions of different FIR structure strategies are assessed and measured by power spectral density (PSD) and bit error rate (BER), and variable MCM plots as well as their potential prototype filters are examined.
\end{abstract}

This is an open access article under the CC BY-SA license.

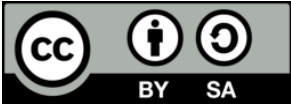

\section{Corresponding Author:}

Ola N. Kadhim

Technical Institute of Al-Mussaib, Al-Furat Al-Awsat Technical University

Najaf, Iraq

Email: ola.najah@atu.edu.iq

\section{INTRODUCTION}

The quick increment of cell phones and the development of new innovations just as administration's request more proficient remote cell systems [1]. The fifth generation (5G) correspondence systems [2], [3] need to help plentiful business situations, for example, internet of vehicles [4], [5], internet of things (IoT) [6]-[10], augmented reality [11], and device-to-gadget interchanges [12]-[14]. Hence, a lot of significant innovations merit concentrating in future correspondence systems, for instance, multicarrier regulation, massive multipleinput multiple-output (MIMO) [15]-[18], and millimeter wave. Because of the basic part of the model channel played in multicarrier balances, that is, it decides the framework execution, for example, stop-band attenuation, inter-symbol interference (ISI), inter-channel interference (ICI) and phase noise results by high operating frequencies, in this different model channel structure strategies in multicarrier correspondence systems are summed up. It is realized in modern digital transmission approaches as the air interface of the fourth generation $(4 \mathrm{G})$ correspondence systems is fit for keeping away from ISI as well as the ICI. The transmitted and detected waveforms are separately produced from inverse fast fourier transform (IFFT) and 
fast fourier transform (FFT), in this manner enormously decreasing the framework multifaceted nature and enhancing the sign transmission rate. OFDM has been utilized related to different innovations, for instance, wavelet OFDM (WOFDM) [19], orthogonal frequency division multiple access (OFDMA) [20]-[22], Alamouti coded OFDM (AC-OFDM) [23], and MIMO-OFDM [15], [24]. In digital signal processing (DSP), digital filtering is one of the usual efficient tools. Despite from the clear advantages of virtually discarding errors in the filter related with passive component variations along temperature, time, op amp drift (active filters), and so on, digital filters are able of performance specifications that would be enormously difficult, if not impossible, to operate with an analog implementation. Furthermore, the characteristics of a digital filter can be simply varying within software control. Therefore, they are widely utilized in adaptive filtering implementation in data communications such as echo cancellation in modems, cancellation of noise, and speech recognition [1], [25]. Although modern digital data transmission has been mostly implemented, it also has the following restrictions: severe out-of-band leakage characteristic; distinct synchronization and orthogonally operation among the subcarriers are required; high peak to average power ratio (PAPR) causing in non-linear distortion of the waveform; sensitive to frequency offset, which has a numerous effect on the system operation [26]. The mentioned situations drive the necessity to show an appropriate modulation scheme in future data communication networks. Tacking in to consideration the requirements of the high spectrum efficiency, high transmission rate and business diversity in the future networks, researchers and technicians in associated fields have making a phenomenal achievement on a number of replaced singlecarrier or multicarrier data transmission modulation schemes [27], including discrete fourier transform (DFT) spread OFDM (CP-DFT-s-OFDM) [15], [28], filter bank multicarrier (FBMC) [29], generalized frequency division multiplexing (GFDM) [30], universal filtered multicarrier (UFMC) [31], and filtered-orthogonal frequency division multiplexing (F-OFDM) [32], [33]. Despite from traditional signal design techniques where signal parameters are obtained by hand, with the improvement of machine learning algorithms, signal structure approaches with data-driven models have attended ascending attention. These modulation signals have their own features and negatives in 5G data communication methodologies [34], respectively. The prototype filter decides the characteristics of a specific modulation signal, thus the application standard of prototype filter is characterized first of all by minimizing the stop-band energy, minimizing the maximum stop-band ripple, minimizing the total interference (ISI and ICI), and so forth.

\section{AIMS AND OBJECTIVES}

The main aims and objectives can be summarized in three basic points: i) Presenting the main drawbacks available in transmitted data signals through a channel; ii) Introducing a new model approach for compensating and enhance the effect of communication channel on transmitted data signal; and iii) Simulating the suggested model using MatLab17b simulation program and comparing the results with previously known topologies.

\section{RESEARCH PAPER ORGANIZATION}

This research paper is organized as the following. Section 4 introduces the theory of digital filtering and windowing techniques used for communication channel realization and shaping. Also, the multicarrier data modulation, with descriptions of their types, and operation techniques has been. Section 5 shows the strategies for structuring and implementing the new proposed approach with illustrations of the resulting outcomes using well shaped figures and tables. Finally, section 6 introduces the description of the proposed approach suggested for cancellation the effects of channel on the transmission data sequence with the influence of the digital filter windowing, the simulation results and available modifications in the suggested approach. The conclusion of the paper declared in section 7 .

\section{DIGITAL FILTERS DESIGN}

The principle of digital filtering can be described as a computation cycle operating to change over a progression of information values that speak to the data waveform through second spoke to by the yield waveform. An advanced digital filter could actualize as programming on a PC or it tends to be equipment that is essential for a particular reason PC. Despite the fact that the computational separating measure is acted in the time space, the depiction of the channel is given in the recurrence area. The real methodology for planning advanced filters has similar essential components as that for simple filters. To start with, the ideal channel reactions are portrayed, and the filter boundaries are then determined. Attributes, for example, adequacy and stage reaction are determined similarly. The key contrast among simple and computerized filters is that as opposed to figuring inductor, capacitor, and resistor esteems for a simple analog filter with coefficient esteems are determined for an advanced filter. Therefore, considering the computerized filter, values supplant the practical resistance and capacitance segments of the simple filter. These values remain in

Digital filters windowing for data transmission enhancement in communication channel (Ola N. Kadhim) 
a register in terms of filter parameters and were utilized with the examined information esteems within the analog-to-digital converter (ADC) to play out the filter computations [1], [16]. The constant computerized digital filter, since it operates in discrete time, process with information in digital form rather than a persistent waveform, and another information point is obtained each inspecting period. In view of this discrete nature, information samples are referred to as numbers, for samples 1 , samples 2 , samples 3 , and so forth. Figure 1 illustrates a low frequency signal consisting of higher frequency noise that should be sifted through. This signal should be converted to digital data via an ADC to deliver tests $x(n)$. The information esteems are taken care of to the computerized filter, and hence a low-pass filter is nominated for this situation. The yield information tests, $y(n)$ are utilized to remake a simple signal utilizing DAC with less glitch. Computerized filters, notwithstanding, cannot be considered as the response to all signal processing filtering prerequisites [35]. So as to keep up constant activity, the DSP processor must have the option to implement all the computations in the filter procedure inside an examining clock period, $1 / f s$ must be done. A quick broadly useful fixed-point DSP, for example, utilizing standard ADSP-2189M kit with 75MIPS can run a total filter tap duplicate gather guidance in 13.3ns. The standard ADSP-2189M kit requires $\mathrm{N}+5$ guidelines for a $\mathrm{N}$-tap filter. For a 100-tap filter, the absolute running time is around $1.4 \mu \mathrm{s}$. This relates to a most extreme conceivable examining recurrence of $714 \mathrm{kHz}$, accordingly restricting the upper sign transfer speed to two or three hundred $\mathrm{kHz}[16]$.

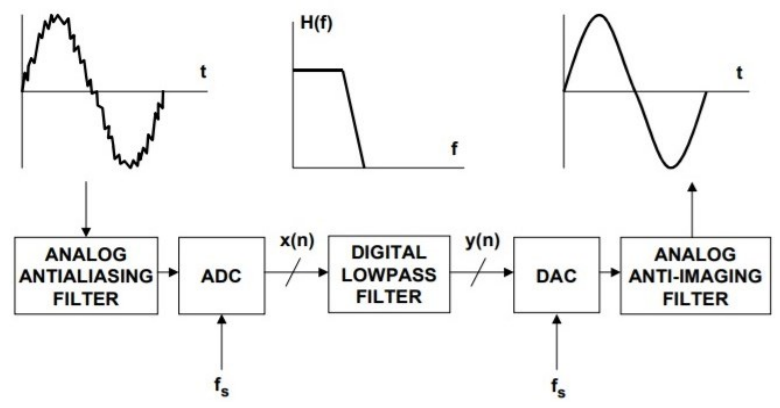

Figure 1. Digital filtering of low frequency waveform and high frequency noise [25]

The most popular filters are specific recurrence devices that communicate specific spectral components with information sign and abandoned the rest. Models have been structured with low pass and band-pass filter. Notwithstanding these overall kinds, the term filters incorporates any framework that plays out some correspondence change through the information waveform [36]. Permanently, utilizing a 3 stages equalizer can't dismiss the spectral components of the information flag yet change the period of the information sign to make up for the attributes of another framework. For instance, consider the examination between a simple and an advanced filter appeared in Figure 2 the cutoff frequency is $1 \mathrm{kHz}$ for the both filters. The simple example filter is acknowledged as a 6-shaft using Type 1 Chebyshev filter (passing wave in pass-band, and suppressing wave in stop-band). Practically speaking, this filter will be acknowledged through utilizing three 2-post arranges, every one of which demands an operation amp, and a few resistances and capacitances. The 6 -shaft configuration is surely not minor, and keeping up the $0.5 \mathrm{~dB}$ wave detail needs exact part choice and coordinating [16].

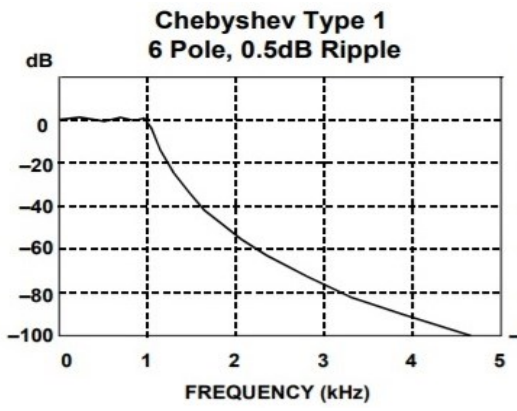

(a)

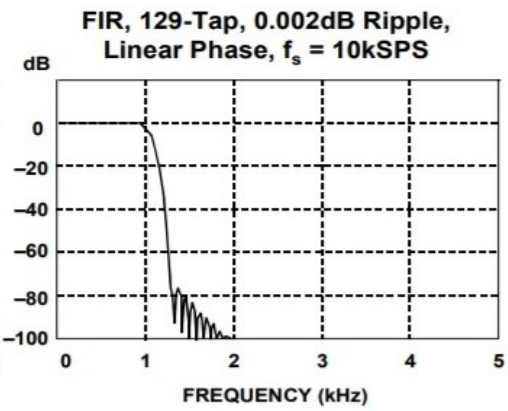

(b)

Figure 2. Characteristics example of (a) analog versus (b) digital filter [16] 
Then again, the advanced finite impulse response (FIR) filter demonstrated has just $0.002 \mathrm{~dB}$ passband swell, straight stage, and a lot more honed move off. Actually, it couldn't be acknowledged utilizing simple procedures. In a pragmatic application, there are numerous different components to consider while assessing simple versus advanced filters. Most present-day signal preparing frameworks utilize a mix of simple and advanced procedures so as to achieve the ideal capacity and exploit the best of both the simple and the computerized world [16].

\subsection{Types of digital filters}

The theory of digital filters can be comprehended by considering these filters as storage models defined with changing stages anchoring on the time field or spectral qualities, their mathematical modeling, and their implementation. Some of the features of a digital filter is the impulse response of the filter which represents the extension of its sequence modeling in the time domain which is called. This trait achieves to two tremendous types [25]: i) the first type known as finite impulse response (FIR) and ii) the second type known as infinite impulse response (IIR) filters.

\subsection{Window function and their equations}

The window strategy, has been defined with a specific transfer function with an ideal low-pass filter utilization, in which a chose window is utilized to accomplish a specific square misfortune. In signal handling, a window work is a numerical capacity with a zero value outside of a chose run. For instance, a capacity that is steady in range and zero is known as a rectangular window that depicts its graphical portrayal shape. In the event that another capacity or waveform/information arrangement is increased by a window work, the consequence of the item is outside the zero territory aside from the part in which they cover, the "glance through the window" [37]. Different window capacities have suggested different basic window capacities are recorded beneath: Rectangular Window, Hanning Window, Hamming Window [16], to lessen changing in the Fourier arrangement strategy, the Fourier parameters are altered by duplicating the boundless drive reaction by a limited weigh grouping $(n)$ known as a window. These windows are described by the size of the fundamental projection, which speaks to the transfer speed among the essential zero negative, the main positive zero, and their frequency proportion. The vitality is profoundly conceivable as it can in the fundamental projection and the vitality in the parallel flap being low as it tends to be. Two wanted properties of a window capacities should be considered in the designing of the window function, the Fourier transformation must have two conditions [16]: i) minimum width in the main lobe and ii) rapidly reduced energy for the Lateral lobe when it reaching zero. The formulation of the window function is written as the following:

$$
(n)=a-(1-a) \cos (2 \pi M n-1), 0 \leq n \leq n-1
$$

$(n)$ is zero elsewhere.

The types of windows utilized in the design the FIR filter is [25]: i) Hamming window, ii) Hammer Window Technology, iii) Hamming's window is shaped like a bell. The window is optimized so that the maximum side lobe is minimized, and iv) Hamming window technology eliminates the single pass-band and stops the band waves. The coefficients of a Hamming window are calculated by comparison.

$$
[n]=0.54-0.46 \cos (2 \pi) x . n . \quad, \quad \mathrm{N}, 0 \leq \mathrm{n} \leq \mathrm{N} 2
$$

\section{DATA CORRECTION PROPOSED SYSTEM}

As recently talked about, there are two crucial kinds of advanced filters: finite impulse response (FIR) and infinite impulse response (IIR). As the phrasing recommends, these arrangements allude to the filter's motivation reaction. By differing the heaviness of the parameters and the filter taps quantity, for all intents and purposes any recurrence reaction trademark can be acknowledged with a FIR channel. As has been appeared, FIR filters can accomplish execution levels which are unrealistic with simple channel methods, (for example, immaculate direct stage reaction). Be that as it may, elite FIR filters for the most part require an enormous number of duplicate gathers and subsequently require quick and productive DSPs. Then again, IIR filters will in general copy the exhibition of customary simple channels and utilize criticism. Subsequently their drive reaction stretches out over an unbounded timeframe. On account of criticism, IIR channels can be executed with less parameters than for a FIR filter. Grid channels are just another approach to actualize either FIR or IIR filters and are regularly utilized in discourse handling utilization. At last, computerized filters loan themselves to versatile sifting utilization essentially due to the speed and simplicity with which the filter attributes can be varied by fluctuating the filter parameters [1], [37], [38]. 


\subsection{Finite impulse response FIR digital filter}

The most rudimentary type of a FIR filter is a moving normal filter as appeared in Figure 3 . Moving average filter are well known for smoothing information, for example, in the investigation of stock costs, and so on. The info tests, $x(n)$ are gone within a progression of support memory taps (marked $\mathrm{z}-1$, relating to $\mathrm{z}$-change portrayal of a defer component). In the model appeared, there are four register taps moving normal. Each example is duplicated by 0.25 , and these outcomes are summed out to result $y(n)$. The figure as well illustrates the overall condition of the digital filter with $\mathrm{N}$ register taps. Also, noting that the quality of the designed digital filter depends on the number of registers taps, $N$.

The moving average approach utilized in the linear filters show that it produced the lowest noise. This is demonstrated in Figure 4 with low noise level results when the sum of register taps is ascended. It can be clearly shown that the pulse response rising period from $0 \%$ to $100 \%$ of is perfectly equal to the total sum of taps of the filter taps times by the sampling time.

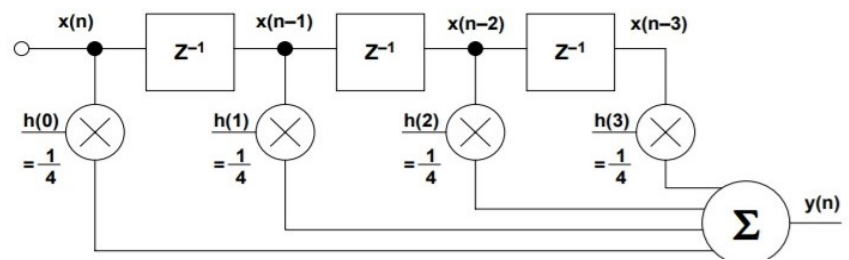

Figure 3. Moving average digital filter with $\mathrm{N}=4$ points [37]

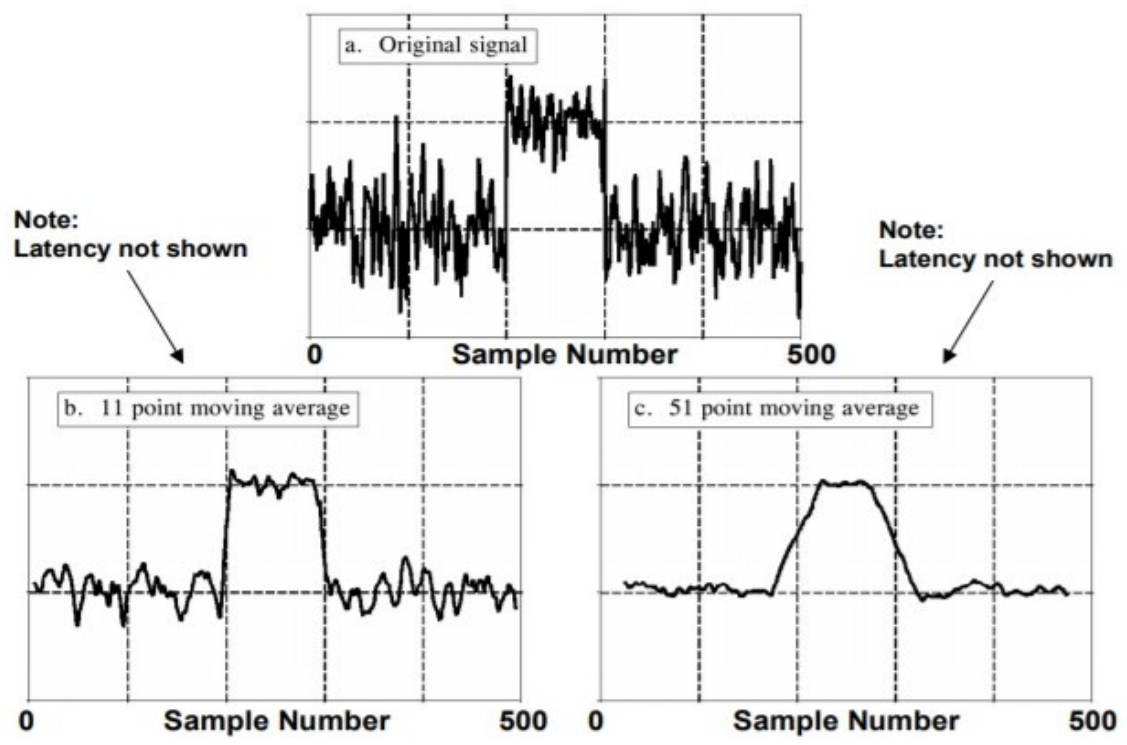

Figure 4. Response of moving average filter under noise effect on step input [1]

Figure 5 shows the moving average filter frequency response which can be formulated with the equation of the form $\sin (x) / x$ increasing of taps number, resulting with more filter sharpens roll off, but controlling to the amplitude of the side lobes. Experimental records showing 14dB side lobes down for the 11 and 31 filter tap. Applications of such filters are usually not preferable with high stop band attenuation. In addition, this technology provides complete compatibility with all types of input signals as well as highlight their importance through the ability to change the number of filter order and increase the number of taps. Figure 6 illustrates the N-tap FIR filter block diagram structure. As has been discussed, an FIR filter must perform the following convolution equation:

$$
y(n)=h(k) * x(n)=\sum_{k=0}^{N-1} h(k) \cdot x(n-k)
$$

where $h(k)$, is the discrete filter transfer function and $x(n-k)$ is the discrete input data sequence to the filter. $\mathrm{N}$, in the (3), denotes the filter taps number and defines its order. 


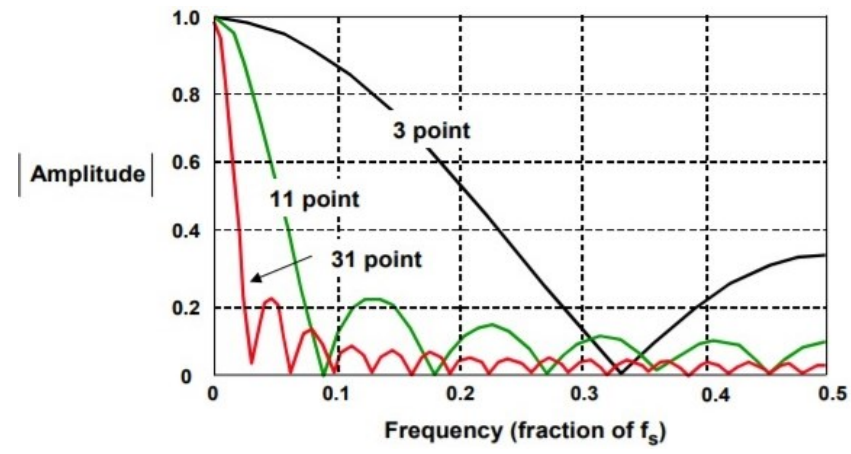

Figure 5. Frequency response of moving average filter [37]

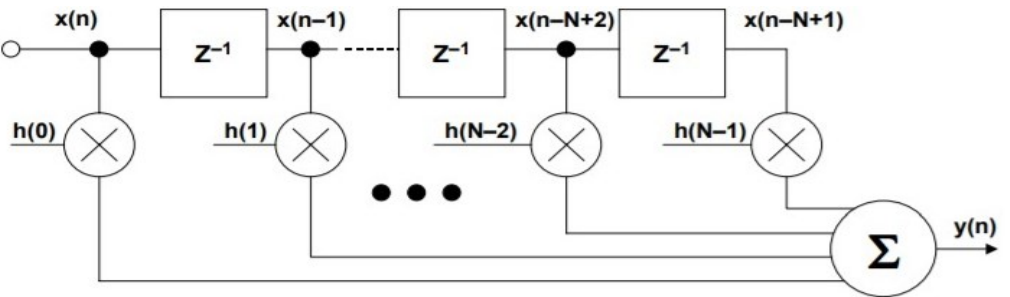

Figure 6. Impulse response of N-tap FIR filter [37]

\subsection{FIR digital filter with windowing}

As mentioned in section 2.1, digital filters with windowing approach begins by representing the transfer function $H(f)$ of the digital filter in mathematical form using Fourier series extension. Coefficients of the designed digital filter have been introduced to represent the system impulse response which will be utilized in the design procedure of the FIR filter transfer function. In any way, the impulse response should be cutting out and applying windowing strategy as discussed in the previous section. When completing the FIR design procedure by cutting out and applying the windowing procedure, the overall system will be transformed in to frequency domain using FFT method. By transforming the FIR filter to frequency domain any frequency range could be selected and hence the digital filter will be ready to perform spectral selection analysis. This facility will be widely utilized in data correction and enhancement field as well as in separation of any unwanted interference signal. Although, the problem of the stop band ambiguity will still existing, but the selectivity and permittivity of the spectrum selection technique introduced by the FIR window design still dominant via the variety of filter window types introduced in literature [25], [39], [40]. Figure 7 Illustrates the frequency response of band pass FIR digital filter with Butterworth window and order of $n=40$ presented by MatLab17b simulation tool box digital filter design. Figure 8 demonstrates the impulse and spectral analysis transfer function of the band pass FIR digital filter with Butterworth window and order of $n=40$ which presented by MatLab17b simulation tool box digital filter design.

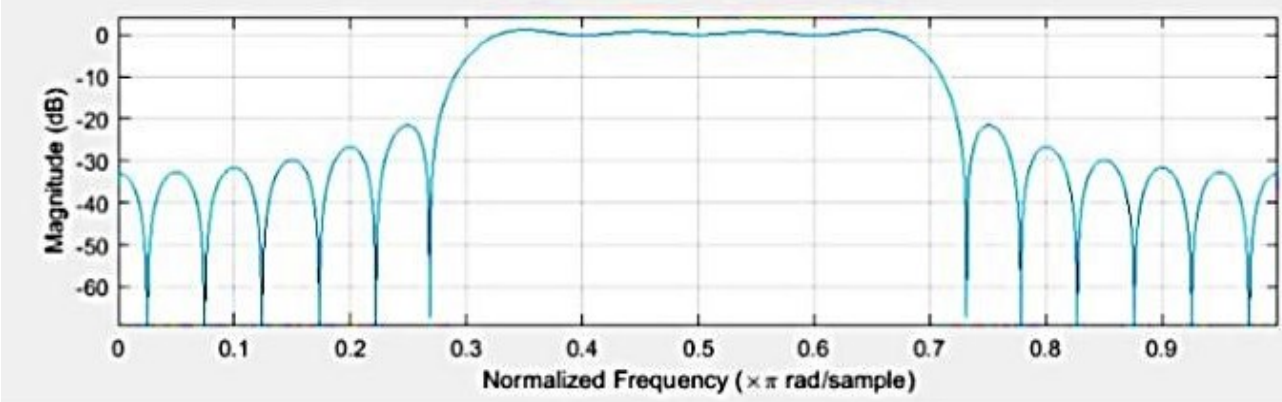

Figure 7. Frequency response of band pass FIR digital filter with Butterworth window, $n=40$ presented by MatLab17b simulation tool box digital filter design 


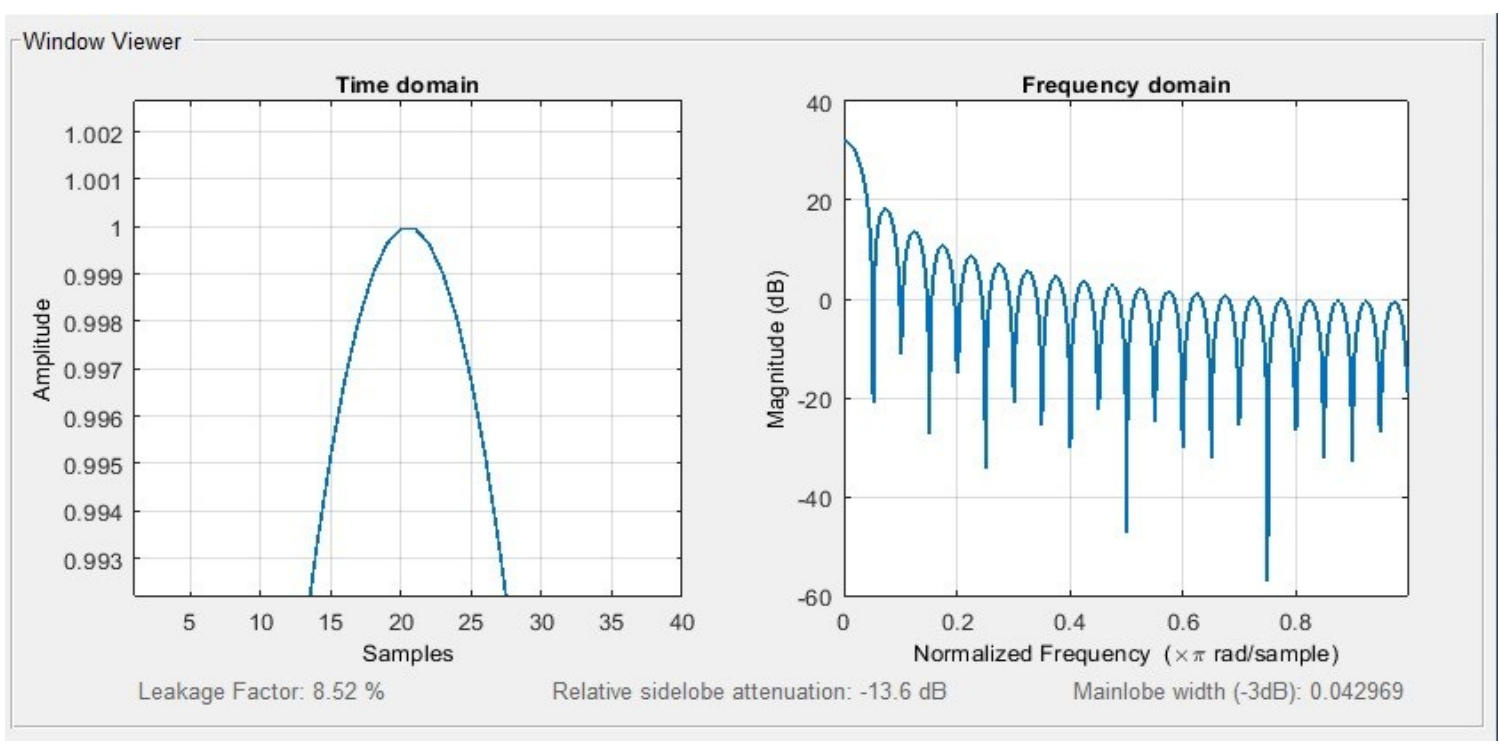

Figure 8. Impulse and frequency response of band pass FIR digital filter with Butterworth window, $\mathrm{n}=40$ presented by MatLab17b simulation tool box digital filter design

\section{PROPOSED SYSTEM DESIGN AND IMPLEMENTATION RESULTS}

In this section, the new proposed data correction system topology has been presented and implemented successfully. The suggested system topology has been simulated using Matlab17b m. files as well as Simulink tool box using the principle of FIR with window approach. The simulated programs have been successfully implemented and tested under the design specifications and the obtained results have been arraigned and presented for discussion. It is obvious and by default that the processing speed of the simulation programs will depend on the design specifications of the utilized P.C. The simulation results from different programs have been analyzed and illustrated in an arraigned manner with suitable analyzing and comprehensive discussion.

\subsection{The new approach system description}

In this research a new modified method is used to enhancing the data transmission communication through a noisy channel. This new modified approach based on influencing the FIR digital filters with window shaping inside the communication system. This influence of the FIR filters will be cancelling the channel noise effect on the transmitted data as well as will reduce the effect of the channel distortion on the information which will more reduce the error in the received data and correct their shapes with enhanced accuracy. Figure 9 show a block diagram of the proposed system.

The implementation of the new approach has been presented with two main programs which have been introduced using MatLab17b simulation program.

a) Data_Enhancement.m.

This program is an $\mathrm{m}$. file MatLab17b code which will show the data transfer of the binary signal 'Data' through a pass band channel with limited band frequency and read in received (data transferred) signal without effect of channel noise the channel design with $n=40$ band pass FIR digital filter with the transmitted data will be enhanced \& corrected using adaptive least mean square least-mean-square (LMS) digital filter.

b) Data_Correction_FIR_Window.slx.

This algorithm is a simulink implementation program that has been used to implement the data transmission communication system with two topologies. The first system topology has been simulated using quadrature phase shift keying, QPSK modem with the present of additive weight Gaussian noise (AWGN) channel to represents the natural noise effect as well as with the Rayleigh channel which represents the effect of the channel noise distortion. The second system topology has been simulated with the introducing of the FIR digital filter with window technique that has been influenced inside the first system topology to eliminate the natural and channel noise effects. Figure 10 demonstrates the overall above mentioned system topology that has been implemented in this research paper. Figures 11 illustrating the first and second system topologies utilized to implement the data correction transmission technique respectively. 


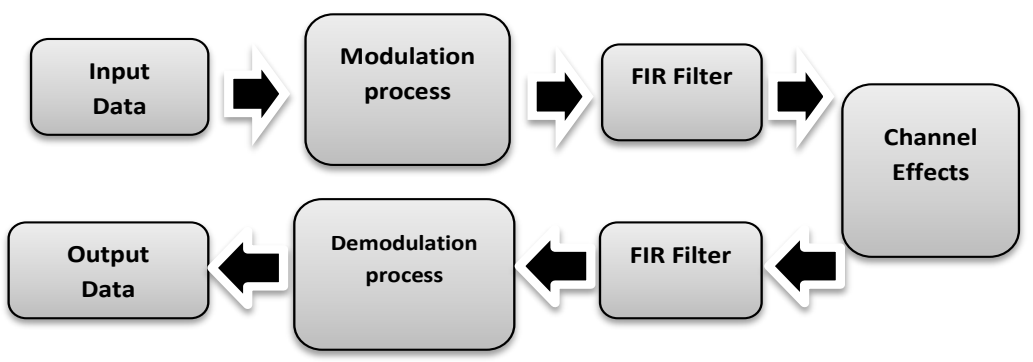

Figure 9. The new proposed system block diagram

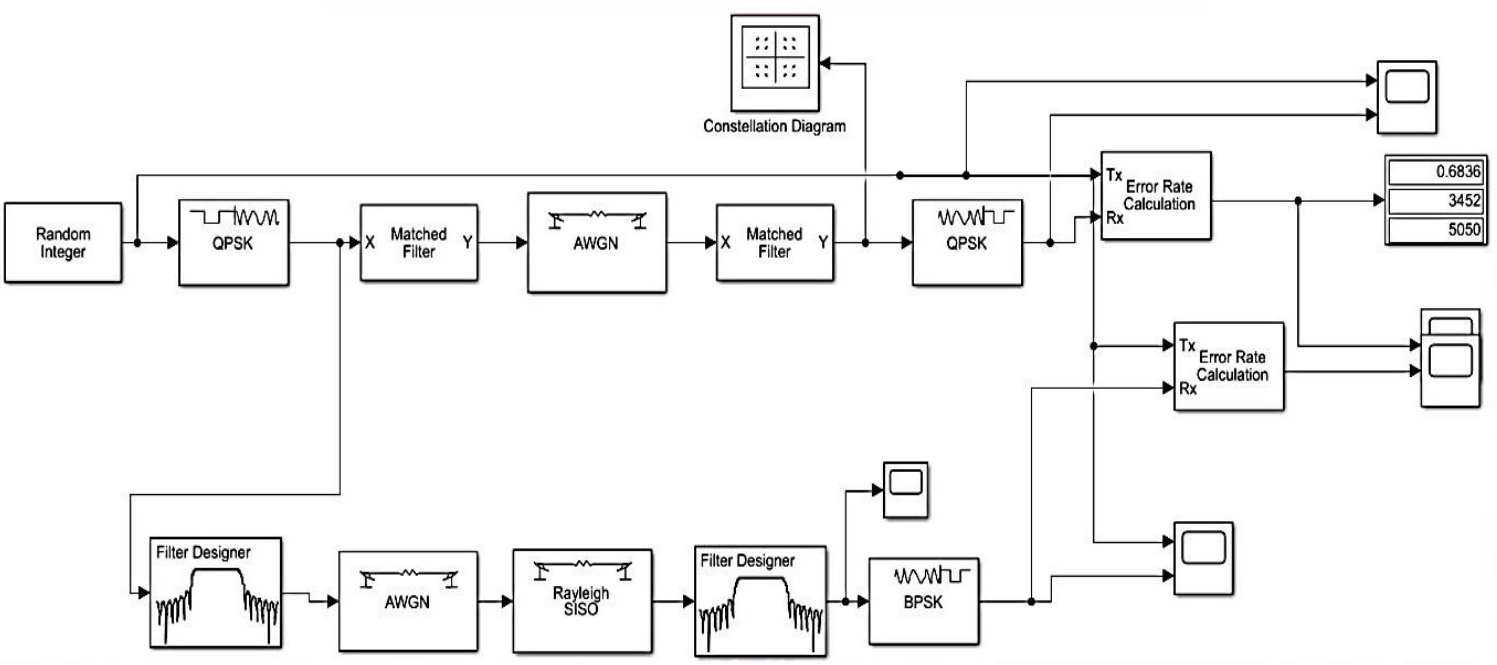

Figure 10. The overall system topology that has been implemented in the research

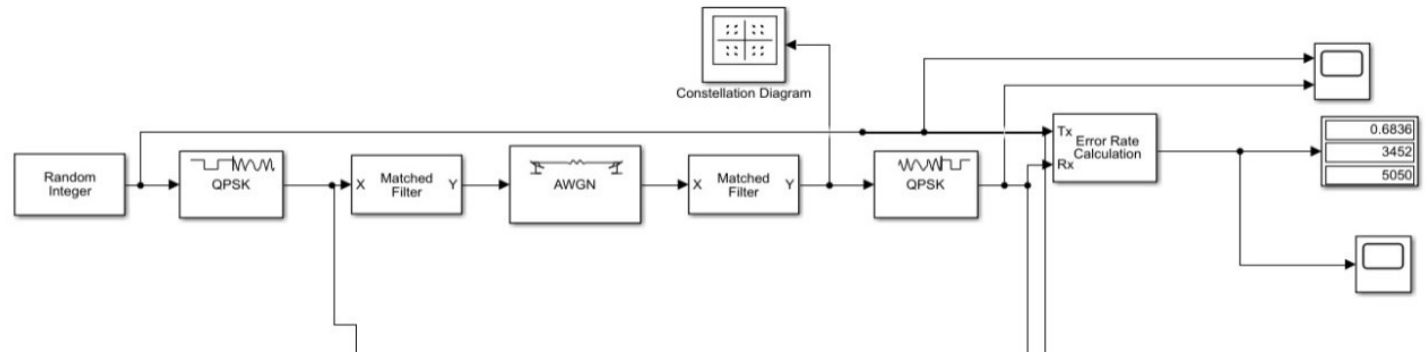

(a)

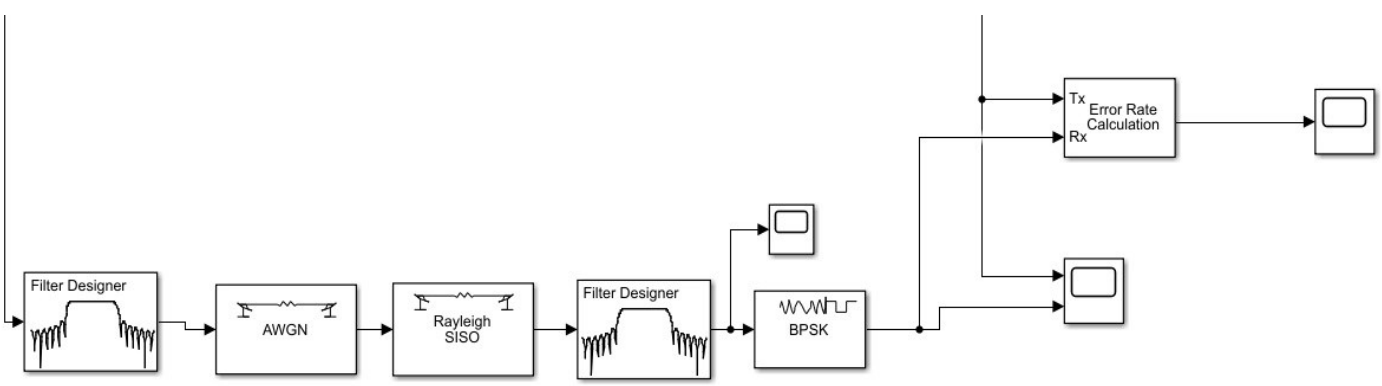

(b)

Figures 11. System topology for data correction transmission technique: (a) first system topology and (b) second system topology 


\subsection{Implementation results}

The first project program has been successfully executed and tested under the design constraints and the test results has been shown in the figures. Figure 12(a) the samples of the input data sequence used to be transmitted via the simulated channel. Figure 12(b) presents the samples of the input data sequence after passing through the simulated BPF channel. The resulting data signal is distorted due to the effect of the channel noise distortion. Figure 12(c) Illustrates the samples of the input data sequence after passing through the estimated LMS BPF Simulated channel. The resulting data signal has the same distortion due to that resulting from the original simulated channel one.

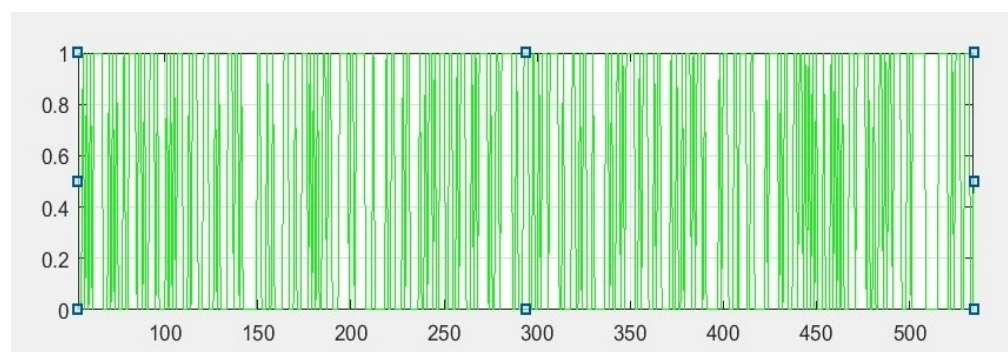

(a)

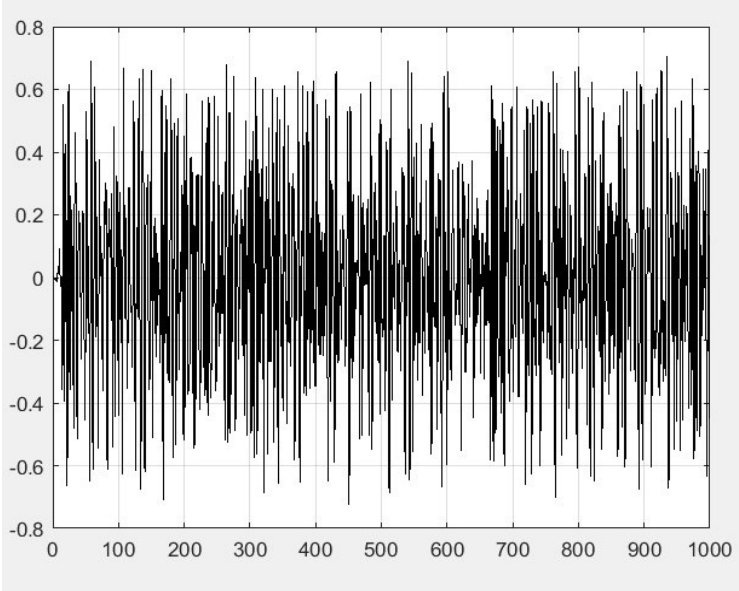

(b)

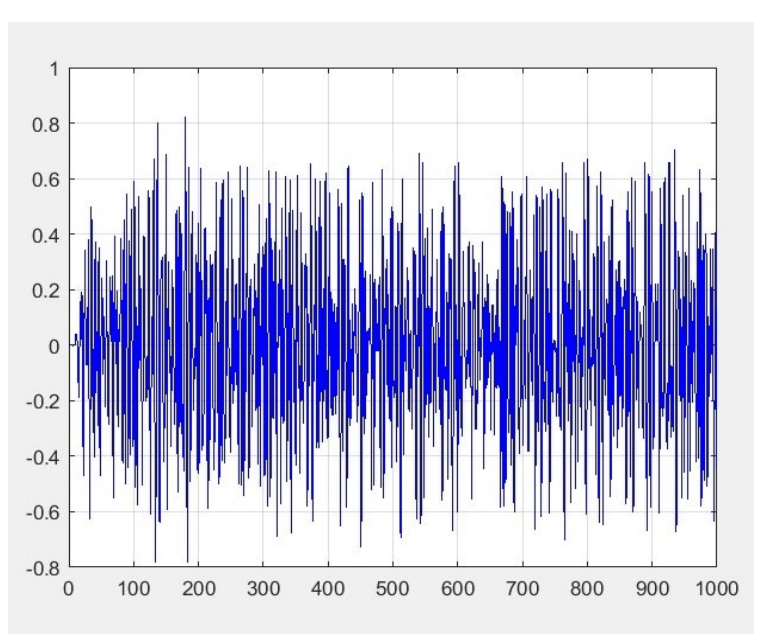

(c)

Figure 12. Data Sequence: (a) input data transmitted sequence channel, (b) input data passing through simulated, and (c) input data passing through estimated LMS simulated channel

By comparing the resulting output data sequences from the original and estimated LMS simulated channels, the resulting error signal will be used to decide the correct design parameters of the simulated noise channel. By implementing this technique, an estimation will be achieved to the simulated noise channel parameters and hence this could be used to eliminate the distortion effect of the transmission channel by implying equalizer procedure. Figures 13 and 14 demonstrating the resulting error sequence signal and the estimated simulated transmission channel impulse response parameters encountered in the program respectively. Furthermore, the estimated channel frequency response has been shown in Figure 15. Now, the second project Simulink program has been successfully executed and tested under the design requirements and the test results has been shown in the figures. Figure 16 shows the resulting spectrum from the random sequence data generator of the first system topology which has been utilized to transmit and receive the data sequence via the AWGN and Rayleigh channels.

After using quadrature phase shift keying (QPSK) demodulator in the first system topology, the received corrupted data sequence spectrum shown in Figure 17 the effect of the AWGN and the Rayleigh channels noise and distortions are obvious on the received data sequence amplitude response spectrum. The received corrupted data signal from the first system topology has been illustrated in Figure 18 together with the transmitted sequence for comparison. The distortion ratio is high with R. M. S. of 0.6877 correction, and the received data sequence is highly distorted and corrupted due to the large value of the channel noise power 
which has been set to $-20 \mathrm{~dB}$ signal-to noise ratio (SNR) under the presence of the AWGN and Rayleigh channels. Also, the error rate calculations curves of the first system topology have been demonstrated in Figure 19 with large R. M. S. error value of 0.6877 .

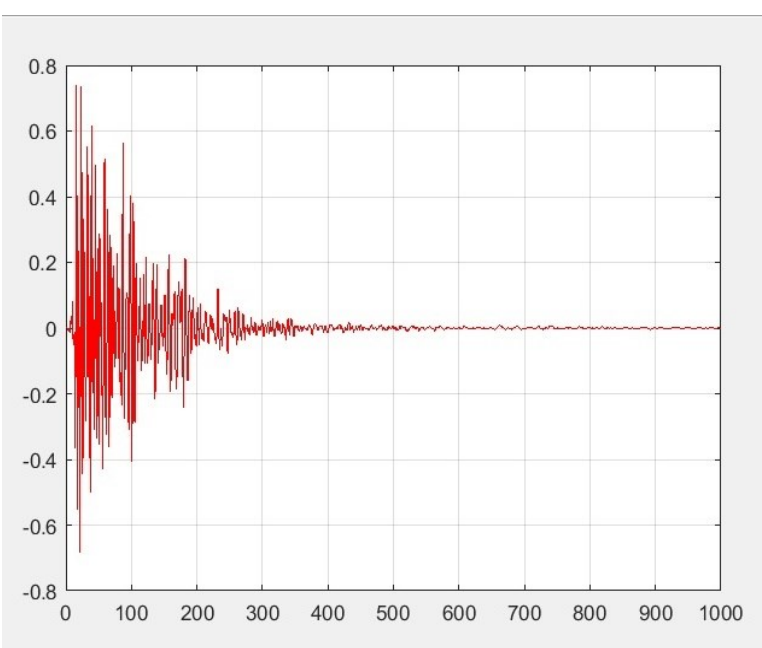

Figures 13. Resulting error signal due to the effect of the estimated LMS simulated channel

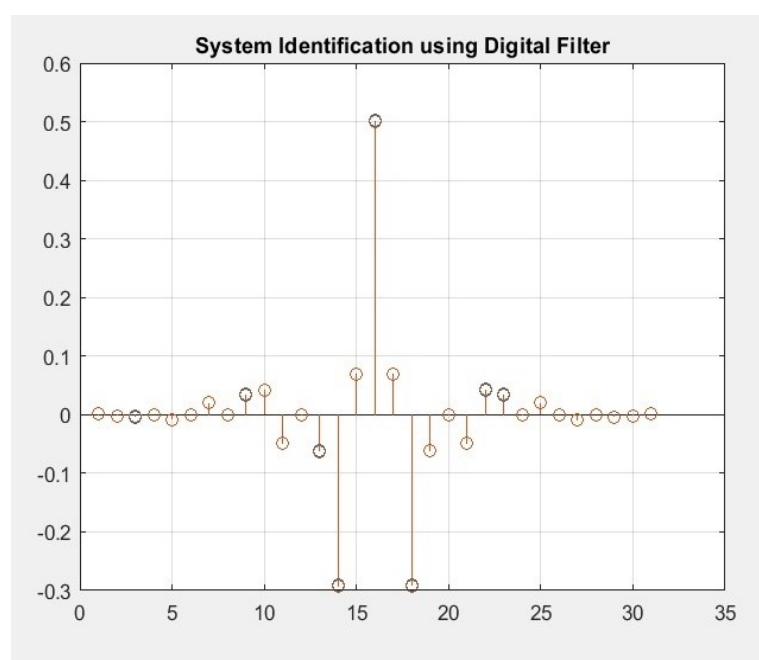

Figures 14. Estimated simulated transmission channel impulse response parameters

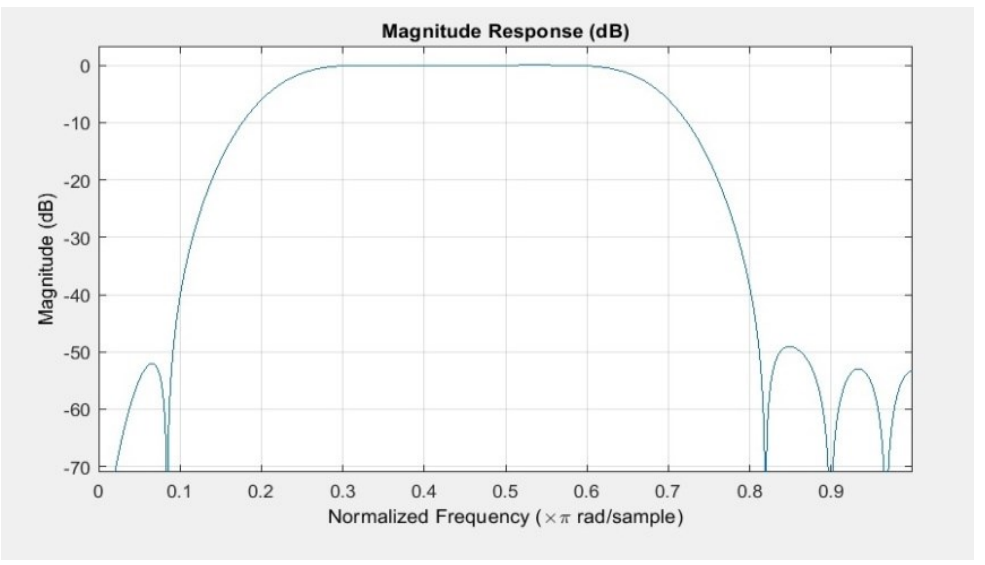

Figures 15. Estimated simulated transmission channel frequency response

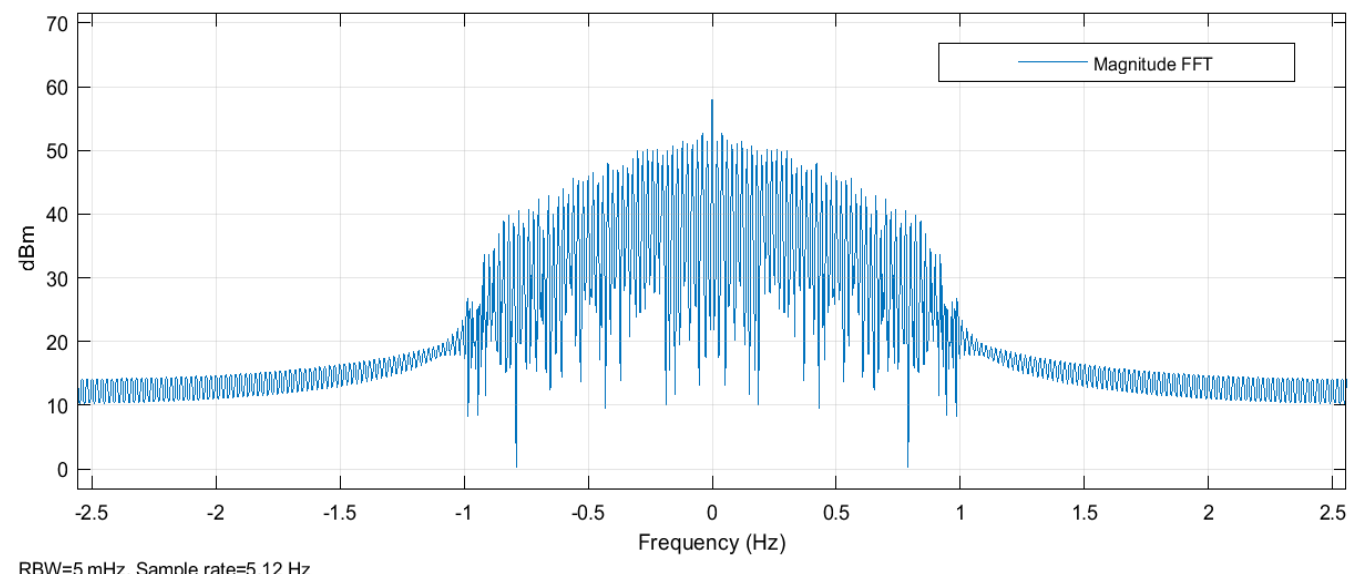

Figure 16. Resulting random sequence data spectrum of the first system topology 


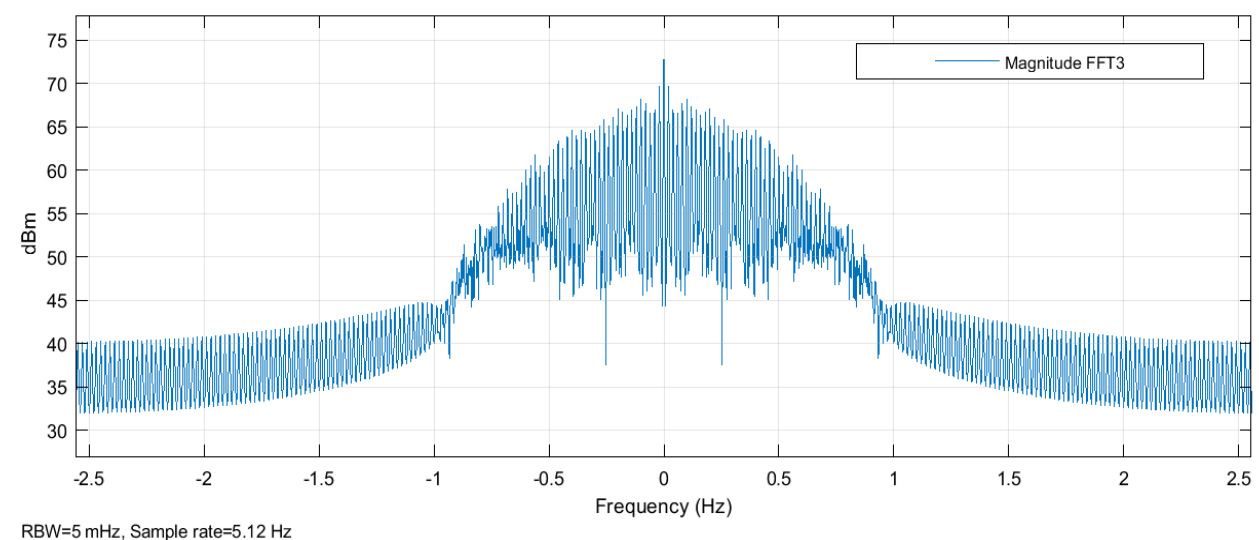

$\mathrm{RBW}=5 \mathrm{mHz}$, Sample rate $=5.12 \mathrm{~Hz}$

Figure 17. Received data spectrum after the QPSK demodulator in the first system topology

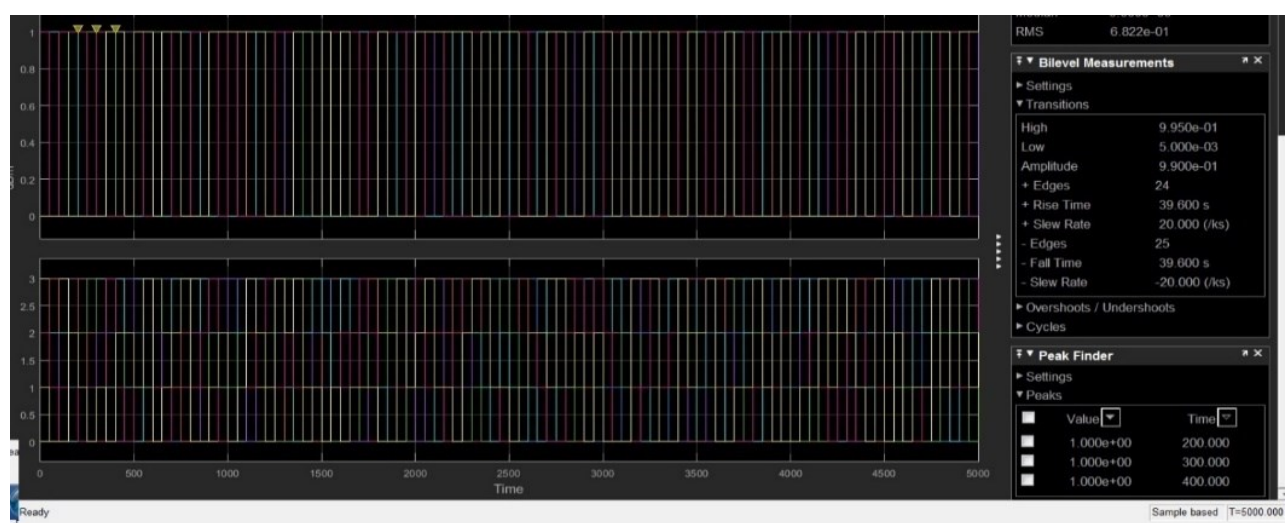

Figure 18. Received corrupted data sequence in time domain

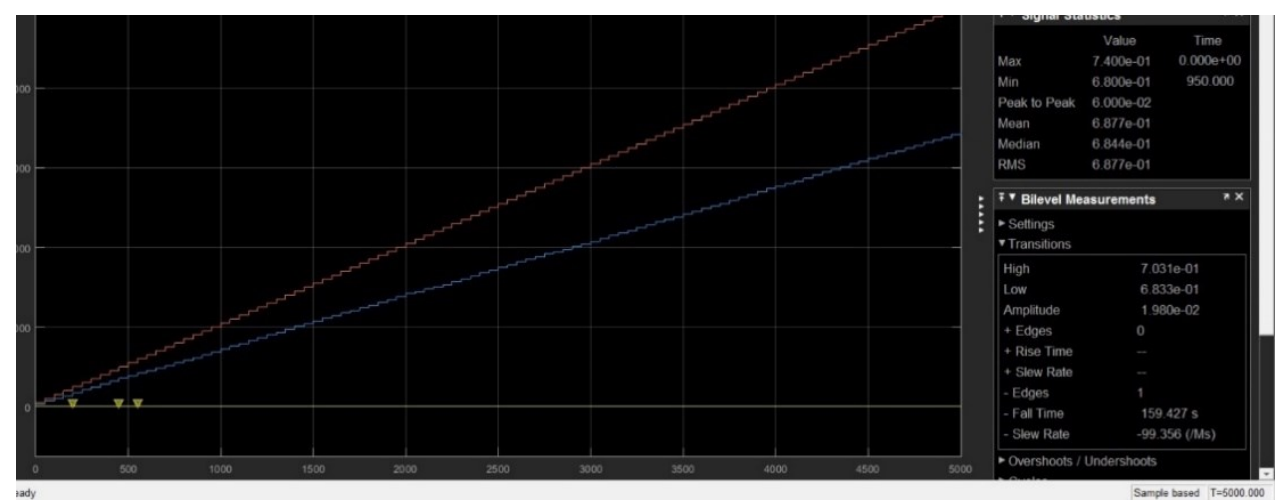

Figure 19. Received error rate calculation curves of the first system topology

The received enhanced data sequence spectrum at the output of the QPSK demodulator in the second system topology, is shown in Figure 20 the effect of the AWGN and the Rayleigh channels noise and distortions are successfully cancelled on the received data sequence amplitude response spectrum as compared to that resulting from the first system in Figure 17. The received enhanced data signal from the second system topology has been shown in Figure 21 together with the transmitted sequence for comparison. The distortion ratio is low with R. M. S. of 0.4719 correction, and the received data sequence is highly enhanced and claret due to the FIR digital filter illumination action to the channel noise which has the same SNR value of $-20 \mathrm{~dB}$ under the presence of the AWGN and Rayleigh channels. Finally, the error rate calculations curves of the second system topology have been demonstrated in Figure 22 with less R. M. S. error value of 0.4719 


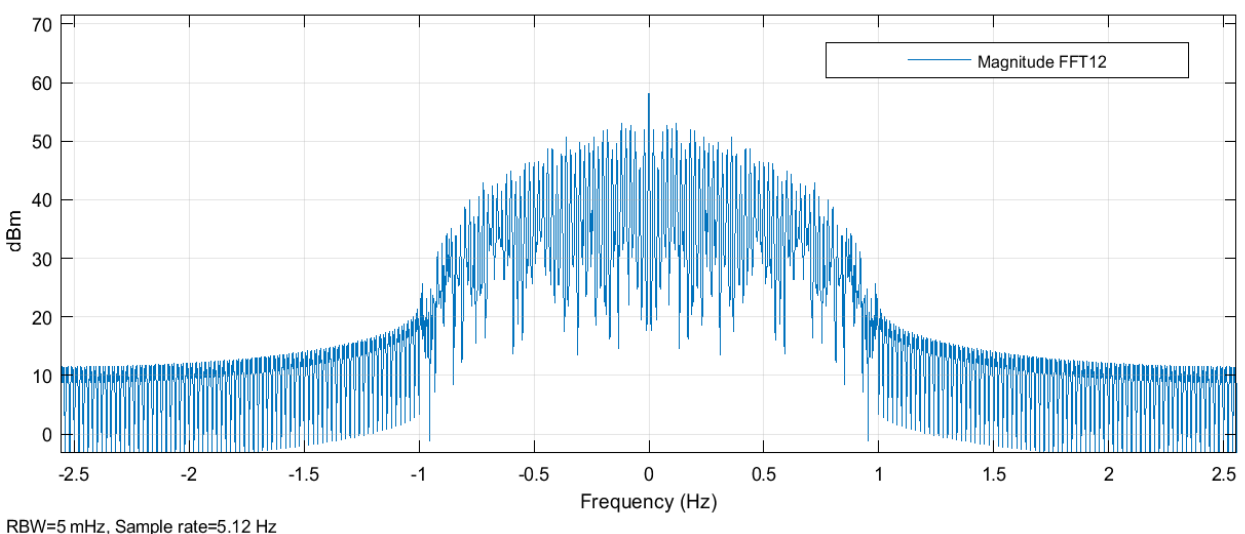

$\mathrm{RBW}=5 \mathrm{mHz}$, Sample rate $=5.12 \mathrm{~Hz}$

Figure 20. Received data spectrum after the QPSK demodulator in the second system topology

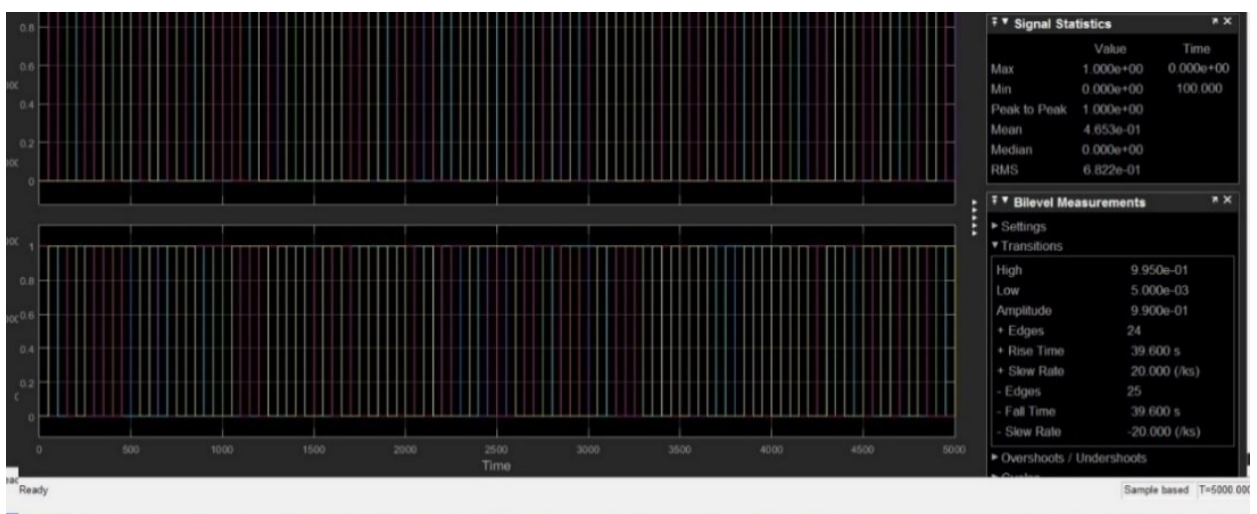

Figure 21. Received enhanced data sequence in time domain

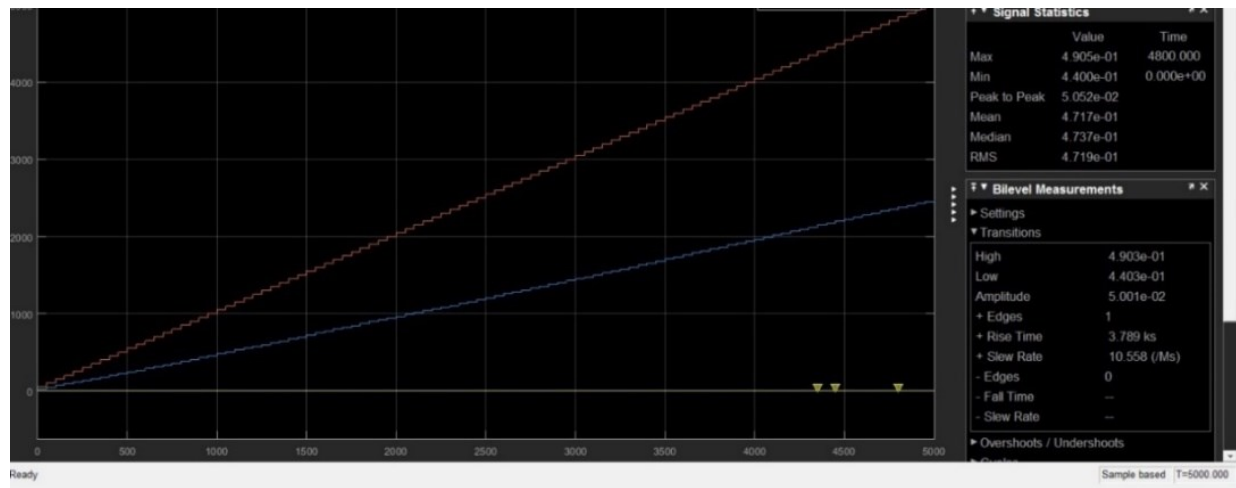

Figure 22. Received error rate calculation curves of the second system topology

\section{CONCLUSIONS}

In this research a new modified approach has been presented for enhancing the data transmission communication through a noisy channel. This new modified approach based on influencing the FIR digital filters with window shaping inside the communication system. This influence of the FIR filters will be cancelling the channel noise effect on the transmitted data as well as will reduce the effect of the channel distortion on the information which will more reduce the error in the received data and correct their shapes with enhanced accuracy. The implementation of the new approach has been presented with two main programs which have been introduced using MatLab17b Simulation program. It is clear from the above spectrum, the guarding technique produced by the second FIR digital filter on the modulated input data sequence from the effect of channel noise. The FIR digital filter will separate the noise distorted spectra from

Digital filters windowing for data transmission enhancement in communication channel (Ola N. Kadhim) 
the modulated data one and this separation will highly increase using window shaping approach. Applying the new topology, the effect of the AWGN and the Rayleigh channels noise and distortions are successfully cancelled on the received data sequence amplitude response spectrum as compared to that resulting from the standard QPSK system. Furthermore, the received enhanced data signal using the new modified approach has been obtained with low distortion ratio with R. M. S. equal to 0.4719 correction. Also, the received data sequence is highly enhanced and claret due to the FIR digital filter illumination action to the channel noise with SNR value of $-20 \mathrm{~dB}$ under the presence of the AWGN and Rayleigh channels.

\section{REFERENCES}

[1] C. B. Rorabaugh, DSP Primer. McGraw-Hill, 1999.

[2] A. F. Demir, M. Elkourdi, M. Ibrahim, and H. Arslan, "Waveform design for 5G and beyond," arXiv preprint arXiv:1902.05999, 2019, doi: 10.1002/9781119333142.ch2.

[3] S. Rosmaniza Ar, N. A. Ahmad, and S. K. Syed Yusof, "Signal modulation techniques in Non-orthogonal waveform for future wireless communication system," Indonesian Journal of Electrical Engineering and Computer Science, vol. 15, no. 3, pp. 1458-1465, 2019, doi: 10.11591/ijeecs.v15.i3.pp1458-1465.

[4] K.-W. Chen et al., "Vision-Based Positioning for Internet-of-Vehicles," IEEE Transactions on Intelligent Transportation Systems, vol. 18, no. 2, pp. 364-376, 2017, doi: 10.1109/tits.2016.2570811.

[5] W. Zhang and X. Xi, "The innovation and development of Internet of Vehicles," China Communications, vol. 13, no. 5, pp. 122-127, 2016, doi: 10.1109/cc.2016.7489980.

[6] D. Pishva, "Internet of Things: Security and privacy issues and possible solution," in 2017 19th International Conference on Advanced Communication Technology (ICACT), 2017, pp. 797-808: IEEE, doi: 10.23919/ICACT.2017.7890229.

[7] I. Yaqoob et al., "Internet of Things Architecture: Recent Advances, Taxonomy, Requirements, and Open Challenges," IEEE Wireless Communications, vol. 24, no. 3, pp. 10-16, 2017, doi: 10.1109/mwc.2017.1600421.

[8] E. Morin, M. Maman, R. Guizzetti, and A. Duda, "Comparison of the Device Lifetime in Wireless Networks for the Internet of Things," IEEE Access, vol. 5, pp. 7097-7114, 2017, doi: 10.1109/access.2017.2688279.

[9] A. Rahman, G. Wu, and A. M. Liton, "Mobile edge computing for internet of things (IoT): security and privacy issues," Indonesian Journal of Electrical Engineering and Computer Science, vol. 18, no. 3, pp. 1486-1493, 2020, doi: 10.11591/ijeecs.v18.i3.pp1486-1493.

[10] A. Rahman, G. Wu, and A. M. Liton, "Mobile edge computing for internet of things (IoT): security and privacy issues," Indonesian Journal of Electrical Engineering and Computer Science, vol. 18, no. 3, pp. 1486-1493, 2020, doi: 10.11591/ijeecs.v18.i3.pp1486-1493.

[11] P. Rosedale, "Virtual Reality: The Next Disruptor: A new kind of worldwide communication," IEEE Consumer Electronics Magazine, vol. 6, no. 1, pp. 48-50, 2017. doi: 10.1109/mce.2016.2614416.

[12] A. Douik, S. Sorour, H. Tembine, T. Y. Al-Naffouri, and M.-S. Alouini, "A Game-Theoretic Framework for Network Coding Based Device-to-Device Communications," IEEE Transactions on Mobile Computing, vol. 16, no. 4, pp. 901-917, 2017, doi: 10.1109/tmc.2016.2586068.

[13] B. Chen, C. Yang, and A. F. Molisch, "Cache-Enabled Device-to-Device Communications: Offloading Gain and Energy Cost," IEEE Transactions on Wireless Communications, vol. 16, no. 7, pp. 4519-4536, 2017, doi: 10.1109/twc.2017.2699631.

[14] M. Haus, M. Waqas, A. Y. Ding, Y. Li, S. Tarkoma, and J. Ott, "Security and Privacy in Device-to-Device (D2D) Communication: A Review," IEEE Communications Surveys \& Tutorials, vol. 19, no. 2, pp. 1054-1079, 2017, doi: $10.1109 /$ comst.2017.2649687.

[15] E. Basar, "On Multiple-Input Multiple-Output OFDM with Index Modulation for Next Generation Wireless Networks," IEEE Transactions on Signal Processing, vol. 64, no. 15, pp. 3868-3878, 2016, doi: 10.1109/tsp.2016.2551687.

[16] Y. M. Tabra and B. Sabbar, "Hybrid MVDR-LMS beamforming for Massive MIMO," Indonesian Journal of Electrical Engineering and Computer Science, vol. 16, no. 2, pp. 715-723, 2019, doi: 10.11591/ijeecs.v16.i2.pp715-723.

[17] M. F. Ismail et al., "45 degree arrangement of compact array antenna for MIMO application," Indonesian Journal of Electrical Engineering and Computer Science, vol. 15, no. 2, pp. 838-844, 2019, doi: 10.11591/ijeecs.v15.i2.pp838-844.

[18] C. S. P and S. C R, "Synthesis of model predictive controller for an identified model of MIMO process," Indonesian Journal of Electrical Engineering and Computer Science, vol. 17, no. 2, pp. 950-956, 2020, doi: 10.11591/ijeecs.v17.i2.pp941-949.

[19] T. Boukour, M. Chennaoui, A. Rivenq, J.-M. Rouvaen, and M. Berbineau, "A new WOFDM design for high data rates in the case of trains communications," in Proceedings of the Fifth IEEE International Symposium on Signal Processing and Information Technology, 2005, pp. 635-638, doi: 10.1109/ISSPIT.2005.1577171.

[20] B. Siva Kumar Reddy, "Orthogonal frequency division multiple access downlink physical layer communication for IEEE 802.16-2009 standard," IET Signal Processing, vol. 10, no. 3, pp. 274-279, 2016, doi: 10.1049/iet-spr.2015.0380.

[21] M. F. Ghanim, "The effect of wavelet transform on OFDM system in modern cellular networks," Indonesian Journal of Electrical Engineering and Computer Science, vol. 15, no. 1, pp. 324-327, 2019, doi: 10.11591/ijeecs.v15.i1.pp324-327. 
[22] V. M. Kulkarni and A. S. Bhalchandra, "Peak-to-average power ratio reduction in wavelet packet modulation Using Binary Phase Sequence," Indonesian Journal of Electrical Engineering and Computer Science, vol. 14, no. 3, pp. 1210-1219, 2019, doi: 10.11591/ijeecs.v14.i3.pp1210-1219.

[23] H. Bao, J. Fang, Z. Chen, H. Li, and S. Li, "An Efficient Bayesian PAPR Reduction Method for OFDM-Based Massive MIMO Systems," IEEE Transactions on Wireless Communications, vol. 15, no. 6, pp. 4183-4195, 2016, doi: $10.1109 /$ twc.2016.2536662.

[24] Y. M. Tabra and B. Sabbar, "Hybrid MVDR-LMS beamforming for Massive MIMO," Indonesian Journal of Electrical Engineering and Computer Science, vol. 16, no. 2, pp. 715-723, 2019, doi: 10.11591/ijeecs.v16.i2.pp715-723.

[25] S. Salivahanan, C. Gnanapriya, and A. Vallavaraj, Digital Signal Processing. Tata McGraw-Hill: second edition, 2010.

[26] A. Sahin, R. Yang, E. Bala, M. C. Beluri, and R. L. Olesen, "Flexible DFT-S-OFDM: Solutions and Challenges," IEEE Communications Magazine, vol. 54, no. 11, pp. 106-112, 2016, doi: 10.1109/mcom. $2016.1600330 \mathrm{~cm}$.

[27] A. Sahin, I. Guvenc and H. Arslan, "A Survey on Multicarrier Communications: Prototype Filters, Lattice Structures, and Implementation Aspects," IEEE Communications Surveys \& Tutorials, vol. 16, no. 3, pp. 1312-1338, 2014, doi: 10.1109/surv.2013.121213.00263.

[28] N. Telagam, U. Somanaidu, M. Arunkumar, M. Sabarimuthu, and N. Kandasamy, "BER Analysis of LTE-OFDM based DWT, Haar transform and Singular Wavelet Decomposition in Stanford University Interim channel," in 2020 Third International Conference on Advances in Electronics, Computers and Communications (ICAECC), 2020, pp. 1-5, doi: 10.1109/ICAECC50550.2020.9339476.

[29] A. Şahin, I. Güvenç and H. Arslan, "A comparative study of FBMC prototype filters in doubly dispersive channels," in 2012 IEEE Globecom Workshops, 2012, pp. 197-203, doi: 10.1109/GLOCOMW.2012.6477569.

[30] H. Shimodaira, A. Sadri, and J. Kim, "Generalized frequency division multiplexing (GFDM) frame strucutre for IEEE 802.11 AY," U.S. Patent 9973364 B2, 2016, May 15, 2018.

[31] V. Vakilian, T. Wild, F. Schaich, S. ten Brink, and J.-F. Frigon, "Universal-filtered multi-carrier technique for wireless systems beyond LTE," in 2013 IEEE Globecom Workshops (GC Wkshps), pp. 223-228, 2013, doi: 10.1109/GLOCOMW.2013.6824990.

[32] H. Poveda, G. Ferré, and E. Grivel, "Way to design an orthogonal frequency-division multiple access-base station receiver disturbed by a narrowband interfering cognitive radio signal," IET Communications, vol. 9, no. 12, pp. 1547-1554, 2015, doi: 10.1049/iet-com.2014.1122.

[33] G. A. Hussain and L. Audah, "BCH codes for 5G wireless communication systems over multipath fading channel," Indonesian Journal of Electrical Engineering and Computer Science, vol. 17, no. 1, pp. 310-316, 2020, doi: 10.11591/ijeecs.v17.i1.pp310-316.

[34] M. Schellmann et al., "FBMC-based air interface for 5G mobile: Challenges and proposed solutions," in 2014 9Th International Conference on Cognitive Radio Oriented Wireless Networks and Communications (CROWNCOM), 2014, pp. 102-107, doi: 10.4108/icst.crowncom.2014.255708.

[35] I. Daubechies, Ten lectures on wavelets. SIAM, 1992.

[36] C. Kim, Y. H. Yun, K. Kim, and J.-Y. Seol, "Introduction to QAM-FBMC: From Waveform Optimization to System Design," IEEE Communications Magazine, vol. 54, no. 11, pp. 66-73, 2016, doi: 10.1109/mcom.2016.1600384cm.

[37] John G. Proakis and D. G. Manolakis, Digital signal Processing. PEAESON, 4Pth edition, 2006.

[38] R. Madasamy and H. Shekhar, "Design of parallel and pipelined DA based OBC fir filter for software defined radio," Indonesian Journal of Electrical Engineering and Computer Science, vol. 14, no. 3, pp. 1228-1234, 2019, doi: 10.11591/ijeecs.v14.i3.pp1228-1234.

[39] A. Sebastian, M. Francis, and A. Mathew, "Non-uniform FIR Digital Filter Bank for Hearing Aid Application Using Frequency Response Masking Technique: A Review," arXiv preprint arXiv:2012.10663, 2020.

[40] G. D. Cain, A. Yardim, and F. J. Harris, "Complex FIR Digital Filter Sharpening With Three-Path Structures," in 2020 28th European Signal Processing Conference (EUSIPCO), 2021, pp. 2324-2328, doi: 10.23919/Eusipco47968.2020.9287393.

\section{BIOGRAPHIES OF AUTHORS}

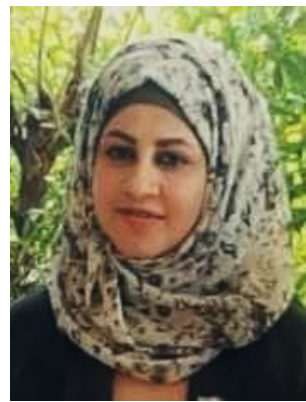

Ola N. Kadhim earned master's degree in computer science from University of Kufa, Iraq in 2019. She earned the B.Sc degree from Babylon University, Iraq 2002. Her interests are digital signal processing, communaction, and image processing. 

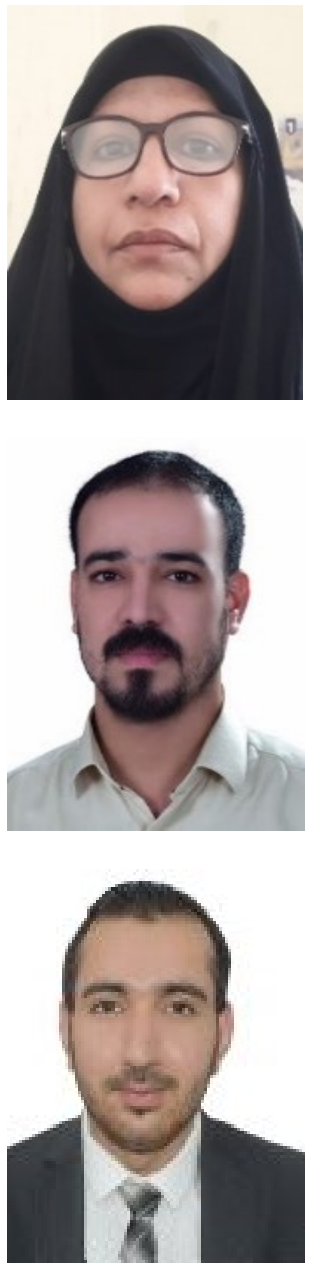

Kifah T. Khudhair earned master's degree in computer science from University of Basra, Iraq in 2008. She earned the B.Sc degree from University of Basra, Iraq 1990. Her interests are image processing, data mining, and object detection.

Fallah H. Najjar earned diploma degree in Computer Systems from Al-Furat Al-Awsat Technical University, Najaf Institute in 2011, B.Sc. degree in Software Engineering from AlImam Ja'afar Al-Sadiq University in 2015, and MSc degree in Computer Science from University of Kufa in 2019. His research interests are artificial intelligence, machine learning, image processing, data mining, computer vision, and data science.

Hassan M. Al-Jawahry earned B.Sc and master's degrees in computer science from University of Kufa, Iraq in 2016, 2019 respectivally. His interests are image processing, data mining, computer vision, and data science. 